\title{
Análisis marginal y estrategia aplicado a una cooperativa de cogestión en Costa Rica
}

\author{
Marginal analysis and strategy applied to a co-managed \\ cooperative in Costa Rica \\ Sergio A. Pineda Campos \\ Universidad Estatal a Distancia (profesor jubilado), Costa Rica \\ spinedacampos@gmail.com \\ Gustavo Hernández Castro \\ Universidad Estatal a Distancia, Costa Rica \\ ghernandezc@uned.ac.cr
}

Recibido: 28/06/2018 • Aceptado: 01/04/2019

\section{RESUMEN}

Son grandes los esfuerzos de los departamentos contables para tener, según la necesidad, los estados financieros periódicos a fin de cumplir con las Normas Internacionales de Información Financiera (NIIF), especialmente, el estado de resultados. Ello cobra vigencia en todo tipo de empresas, aunque con mayor requerimiento en empresas medianas y grandes, o bien en organizaciones diversificadas, en las cuales hay resultados por división, por gerencias, por puntos de venta, por líneas de negocios y categorías de productos, entre otros. El problema de las Cooperativas a resolver es la insuficiente información financiera para la toma de decisiones, especialmente las relacionadas con costos, cadena de valor, operaciones, inversión y financiamiento. El aporte del análisis marginal ha sido bien documentado, no así su visión desde la gerencia estratégica. Este artículo pretende enmarcar el aporte del análisis marginal

Sergio A. Pineda Campos • Gustavo Hernández Castro 
como un objetivo estratégico del valor del negocio aplicado a una cooperativa de Cogestión de productos cárnicos.

Palabras clave: Costes; eficiencia; parámetros marginales; estrategia; valor del negocio.

\begin{abstract}
Accounting departments make strong efforts to have periodic financial statements whenever necessary to comply with the International Financial Reporting Standards (IFRS), especially profit and loss statements. This is true for all types of companies, although requirements are more complex in medium and large companies, or in diversified organizations, in which results are stated by division, management offices, points of sale, business lines and product categories, among others. Cooperatives must solve the problems they have, related to insufficient financial information for decision making, especially information related to costs, value chains, operations, investment and financing. The contribution of marginal analysis in this context has been well documented, but not from the perspective of strategic management. This article seeks to illustrate the contribution of marginal analysis as a strategic objective of business value applied to a co-managed cooperative of meat products.
\end{abstract}

Keywords: Cost; efficiency; marginal parameters; strategy; business value.

\title{
INTRODUCCIÓN
}

El presente artículo es el resultado de una investigación realizada en una cooperativa de cogestión que procesa productos cárnicos. Se analiza la relación existente entre el análisis marginal y la gerencia estratégica como dos elementos complementarios de la disciplina administrativa moderna. Todas las organizaciones, indistintamente de su tamaño, deben de considerar su importancia para la formulación de la estrategia empresarial y, con ello, la generación de empleo (Hernández, 2015).

El reto de las empresas es hacer que el análisis marginal sea una variable que esté contemplada dentro de los planes organizacionales, no como un simple indicador para los análisis de resultados financieros, sino como 
parte integral de todos los elementos que conforman el giro comercial de una compañía; en consecuencia, el análisis marginal, debe considerarse desde la estrategia misma de la organización. El concepto de estrategia tiene como punto de partida la formulación de tres principios rectores: saber qué se quiere, querer hacerlo y trabajar en ello o llevar las ideas a la práctica. Aunque dichos principios parecieran sencillos, se requiere un método para aplicarlos a la realidad organizacional. A esta metodología se le denomina gerencia estratégica (Hernández, 2013).

La gerencia estratégica está vinculada al proceso administrativo: planear, organizar, dirigir y controlar. Para efectos del tema que ocupa este trabajo, se adiciona un elemento indispensable para el proceso: el análisis marginal como insumo de salida y entrada para analizar en términos económicos el valor del negocio (Griffin, 2011). Establecida esta vinculación, se define la gerencia estratégica como el proceso mediante el cual los diferentes niveles de la organización se ajustan para que las acciones emprendidas (actividades, productos y servicios) contribuyan a potenciar las capacidades empresariales que abarcan desde el talento humano hasta el rendimiento incremental de todos los activos de la compañía (David y David, 2017).

Saber qué se quiere es planear. Es el esfuerzo de un equipo humano para formular y alcanzar los objetivos propuestos. Comprende la toma de decisiones porque supone que hay que elegir entre todas o varias alternativas posibles para llegar con éxito al futuro proyectado por la compañía (Drucker, 2004). Una vez definido lo que se quiere hacer, el paso siguiente en la gerencia estratégica es querer hacerlo. Bajo este principio, se deben considerar las variables de organización, dirección y control. Finalmente, trabajar en ello es llevar las ideas a la práctica. Equivale a que la totalidad de la organización esté involucrada en el proceso de gestión del valor del negocio y en la cual la competitividad y rentabilidad de la empresa no debe de sustraerse de la filosofía empresarial de la organización. Lo anterior porque las compañías se gestan con el objetivo de obtener utilidades. Una de las formas de medir la competitividad y la rentabilidad es a través del análisis marginal; en este sentido, las organizaciones de emprendimiento cooperativo, del cual tratan éstas páginas, deben incorporar este valor cuantitativo en la planificación y gerencia estratégica para gestionar el valor del negocio (Porter, 2009; David y David, 2017). 
Así pues, como parte de la evaluación de la información contable, especialmente para el ejercicio periódico del análisis financiero, aparece con mucha frecuencia el estado de resultados. Según el interés de quien administra, este puede requerir de datos o información mensual, trimestral, semestral, o bien, según las necesidades organizacionales, de forma anual, pues acompaña a los demás estados contables. Cabe mencionar la vinculación existente de la gerencia como herramienta de análisis estratégico en las cooperativas y en el sector de la economía social (Sanchis y Campos, 2006).

En empresas cuyo nivel transaccional, diversificación o tamaño involucra el procesamiento y la evaluación sistemática de resultados (en la cual la informática para la toma de decisiones es una herramienta común en nuestros días), suele suceder que se esperan los datos de resultados pertenecientes a la semana o el mes. Estos confirman las estrategias de ventas diseñadas, la gestión de cobros, la satisfacción general del cliente con el producto o servicio; en fin, una serie de variables que son ajustables o que, debido a los resultados, pueden ser objeto de un replanteamiento y cambio de estrategia (Thomas y Hunger, 2013).

El esfuerzo de las áreas contables para ajustar, procesar y obtener la información que se refleja en el estado de resultados es complejo. Algunos ejecutivos, al tener la información en pantalla, buscan en primera instancia si hubo pérdida o utilidad, así como el porcentaje correspondiente. Luego, pueden empezar a analizar los componentes básicos del estado, las ventas y el costo de estas, que se vería reflejado en los ingresos después de ventas; analizan los costos y gastos totales y, adicionalmente, realizan comparaciones con periodos similares para obtener así algunas conclusiones. En este contexto, este artículo tiene el objetivo de señalar las ventajas del análisis marginal a la interpretación de los estados de resultados como una metodología distinta e innovadora respecto a los tradicionales modelos de interpretación de estados para la toma de decisiones desde la gerencia estratégica (Gitman, Berk, DeMarzo, y Benjamin, 2018).

Amaro (2012) propone el estudio del análisis marginal para la toma de decisiones sobre costos y precios. Por su parte, Mankiw (2015) explica el análisis costo-beneficio; Rufasto (2007), desde la ingeniería de procesos, establece que es posible contar con la colaboración de herramientas marginales, especialmente para cuantificar la productividad, entre otros 
aspectos relacionados: indicadores de rendimientos marginales de productividad. En tanto que Cohen (2011), establece que el "análisis marginal estudia la generación de utilidades en la empresa, en función de los aportes individuales de cada tipo de bien o servicio producido" (p.1).

Asimismo, Cohen (2011), define el análisis marginal de la siguiente forma “(...) El análisis marginal estudia el aporte de cada producto/servicio/cliente a las utilidades de la empresa. Permite, por ejemplo, contestar las siguientes preguntas: ¿A partir de qué volumen mínimo de ventas conviene lanzar un nuevo producto?, ¿Conviene dejar de producir un determinado producto existente?, ¿Realmente le conviene a la empresa estar atendiendo a un determinado cliente?, ¿Convendría cerrar directamente una fábrica o sucursal?, ¿Cuánto tengo que vender para que convenga continuar? (Lambreton y Garza, 2016).

Para realizar el análisis financiero de los estados de resultados se efectuó una búsqueda de fuentes representativa. Dicha práctica es generalmente aceptada con raíces teóricas de una gran variedad de autores, pero muy especialmente, en lo que respecta a su aplicación, cada profesional vinculado a este tipo de análisis, adapta la correlación de datos según sea su necesidad. Técnicas como la comparación entre periodos inmediatos anteriores, periodos iguales de años diferentes, análisis vertical y horizontal, presentados en cifras relativas o absolutas, así como otras formas de obtención de información para la toma de decisiones, resultan habituales en el ejercicio profesional. Igualmente, se podrían citar los trabajos de Weston y Brygham (1994). También, y no menos importante dentro de los ejemplos de autores que tratan sobre los temas de análisis financiero, vinculados con el planeamiento estratégico y los costos gerenciales, no sería posible dejar de mencionar a Ross, Westerfiel y Bradford (2010); Hardford, Berk y DeMarzo (2010); Gitman (2010); Gomero y Montes (2011) y Golpe (2016).

Evans (2017) enfoca el análisis marginal desde la perspectiva para calcular las tasas marginales de retorno entre tecnologías (de una tecnología de bajo costo a una tecnología de costo mayor). El principio económico de este análisis beneficia a los tomadores de decisiones para invertir hasta donde el retorno de cada unidad extra invertida sea igual a su costo.

Independientemente del tipo de organización (cooperativas, ONG, privadas, públicas o mixtas), el profesional contable y especialmente el 
financiero, emplean con mayor o menor énfasis las diferentes herramientas de análisis financiero que la teoría o el conocimiento de la materia puede ofrecer. Sin embargo, el fin primordial del esfuerzo intelectual y técnico del analista contable-financiero se encuentra dirigido a la obtención de información relevante que les permita a los tomadores de decisiones, la adopción de medidas que maximicen la utilidad.

El análisis se hace mediante el estudio de cambios o variaciones, utilizando relaciones absolutas y porcentuales en momentos diferentes del tiempo, donde los resultados obtenidos pueden interpretarse con sus respectivas tendencias y efectos, obteniendo conclusiones en la forma de información pertinente. Así, se puede citar el análisis vertical y el análisis horizontal, como representantes de un proceso de diagnóstico -entre otros- de uso más extendido y al que se hace referencia en la parte final de este documento.

Por otro lado, la utilización del análisis mediante razones financieras corrobora y cuantifica con puntualidad los efectos que pudieron detectarse en el análisis horizontal y vertical, con la posibilidad de establecer relaciones en diferentes temas financieros y así concretar el desempeño real de la empresa. Cada razón vincula al menos dos partidas de los estados financieros, las cuales guardan una relación lógica y coherente, donde cada resultado es una medida para una situación específica. El balance general y el estado de resultados conforman la base del análisis de razones, permitiendo que el analista seleccione las razones de las más usadas, así como la adaptación de esquemas de índices por temática o mero interés (Hardford, Berk y DeMarzo, 2010).

Dependiendo del o los autores que aborden la clasificación de las razones financieras, estas pueden clasificarse en razones de estabilidad, razones de gestión y razones de rentabilidad. Los resultados de las razones financieras, en su conjunto, le permiten al analista la comparación histórica, o bien, la comparación con indicadores de la industria en la que la empresa participa. Todo ello dependerá de la memoria organizacional o de la publicación y sistematización de la información de las empresas que conforman las industrias y sus sectores.

En relación con el análisis dirigido al rendimiento sobre los activos, el sistema Du Pont de análisis financiero aborda más puntualmente el rendimiento 
de operación desde dos componentes: la rotación del activo total y el margen de utilidad de operación. Por otra parte, el apalancamiento financiero termina por favorecer un panorama más concreto sobre la manera en la que la empresa obtiene su rentabilidad, estableciendo premisas sobre el riesgo o riesgos asociados (Gitman, Berk, DeMarzo, y Benjamin, 2018).

El profesional contable-financiero tiene a su disposición estas y otras variantes en cuanto al análisis financiero, las cuales debe ajustar a las necesidades propias de la organización, de la actividad y a la conveniencia del objetivo que persiga. Así las cosas, las oportunidades y alternativas se manifiestan abiertas para el desarrollo de esfuerzos novedosos e integrales que permitan la captación de información necesaria para una efectiva toma de decisiones.

\section{METODOLOGÍA}

Esta investigación es de corte mixta (Cortés e Iglesias, 2005). El estudio recoge y analiza la postura del emprendedor cooperativo analizado. Durante la etapa de la recolección de la información se combinaron instrumentos del enfoque cualitativo y cuantitativo, como lo son: la aplicación del método Delphi, cuadros estadísticos, cuestionarios, entrevistas en profundidad y observación participativa, lo cual ha permitido enmarcar el proceso dentro del enfoque mixto. Se procedió al análisis de las variables con el propósito de identificar las subyacentes, o bien, que explicaran la configuración de correlaciones dentro de las variables observadas. Estas explican y tipifican el modelo de organización y la operación del emprendimiento cooperativo en tres componentes a saber: Factor económico (análisis marginal), Planeación estratégica, Externalidades cooperativas positivas (Kline, 1998; Hiller \& Hiller, 2008)

El análisis factorial o reducción de factores se obtiene por ciento de la varianza total. De tal manera que el porcentaje obtenido brinda un valor el cual determina si es el análisis es válido o no. Se consideran válidos los resultados si los factores explican al menos $95 \%$ en las ciencias naturales. Para el caso de esta investigación y de las ciencias sociales es frecuente un resultado del $60 \%$ al $65 \%$ del total de la varianza.

Para tal fin, se desarrolló un modelo matemático para la interpretación de los estados de resultados aplicable en primera instancia (validación) a una 
empresa cooperativa de cogestión de productos cárnicos en Costa Rica, con una alta diversificación (líneas de producción disímiles y, por ende, múltiple variedad de productos) dirigida a diferentes mercados: Nicaragua, Guatemala, México, CARICOM (Comunidad del Caribe), Puerto Rico, Estados Unidos, China y Japón, entre otros.

La empresa cooperativa mantiene un alto volumen de inventario de materias primas, en proceso y con periodos de tiempo estrechos para su comercialización, ya que $90 \%$ de la producción son productos perecederos. La cooperativa procesaba mensualmente más de siete mil reses y cerdos, con el fin de abastecer al mercado de carnes frescas y preparadas de alta calidad nutricional.

Cuenta con una plantilla de personal de alrededor de 850 colaboradores, la cual puede fluctuar en relación con el ciclo de producción y comercialización nacional y de exportación que presentan los mercados. Su Consejo Directivo está conformado por los sectores productivos y regionales del país, que están asociados a la cooperativa. También, hay representación en el Consejo del sector trabajador de la empresa, como asociados al modelo de cogestión escogido en su día.

Desde hace 15 años, la dirección de la empresa decidió combinar dos formas de comercialización de sus productos cárnicos a nivel nacional. En principio, mantener el modelo tradicional de "carnicería" a granel o al detalle y, por otro lado, incursionar en un concepto comercial de "centros de carnes", donde el uso más especializado de tipos de cortes de carnes, el empaque al vacío, la presentación al cliente y la complementariedad con otros productos afines, forma parte de la estrategia empresarial.

En el corto plazo, se proyecta la incursión del modelo de "centros de carnes" bajo un esquema de "franquicia". Sin embargo, se encuentra aún a la espera de nuevas experiencias internas y de diagnósticos más actualizados para que el Consejo Directivo tome las decisiones pertinentes.

Como primer paso, se desarrolló un modelo utilizando el análisis marginal para establecer la rentabilidad y conveniencia de mantener o cerrar puntos de venta; posteriormente, se amplió el modelo matemático para realizar una investigación formal. Después de revisar una serie de modelos matemáticos y teorías para la interpretación de los estados de resultados, 
se llegó a determinar que era necesario desarrollar un modelo de análisis más funcional, resumido y eficiente desde la perspectiva, inclusive, de la informática.

La revisión de la teoría existente involucró dos grandes fines. El primero fue la recopilación de elementos temáticos referenciales que contribuyeran a determinar que el modelo matemático propuesto es innovador, puesto que no se encontró en la bibliografía estudiada la relación de las dos teorías (análisis marginal e interpretación de estados financieros). En segundo lugar, el modelo matemático de análisis marginal diseñado se validó para establecer los alcances respecto a los modelos tradicionales de interpretación de estados de resultados.

Es menester aclarar que, a solicitud del representante legal de la Cooperativa de Cogestión investigada, se omite el nombre jurídico de esta y solamente se menciona la actividad económica que realiza el emprendimiento cooperativo, debiendo utilizar datos financieros aproximados de la división cárnica de la cooperativa para la correspondiente demostración de la identidad matemática.

Asimismo, los investigadores dejan abierta la posibilidad a los interesados en la temática, de solicitar información de la base de datos disponible, que sustenta las variables estadísticas, matemáticas, así como resultados de entrevistas y de los grupos focales, que alimentaron el desarrollo y las posteriores conclusiones que arrojó la pesquisa.

Para la elaboración del modelo matemático propuesto y su validación respectiva se acudió a dos teorías. En primer lugar, la interpretación tradicional y aceptada de estados de resultados; en segundo lugar, lo que respecta al análisis marginal, el cual se encuentra en diferentes áreas del conocimiento. La aplicación investigativa demandó un desarrollo conceptual y de utilización lógica-matemática mediante la correlación de datos. Por otro lado, fue necesario describir las dos teorías estudiadas, de tal manera que se logre el pleno convencimiento de sus bondades particulares (Pineda, 2007).

Para efectos de esta investigación, la aplicación de la técnica Delphi para validar el modelo matemático allanó la principal preocupación metodológica. Esta técnica se aplicó a un panel de expertos (personas ajenas a 
la cooperativa seleccionada) en los temas de análisis marginal y análisis financiero. El objetivo fue obtener un consenso de criterio especialista respecto al tema investigado. De las ventajas de la aplicación de la técnica Delphi se destacan el anonimato, la retroalimentación controlada por los investigadores y la respuesta estadística de grupo (Landeta, 2002; Cortés e Iglesias, 2005; Cabrero e Infante, 2014; Campos, Melián y Sanchis, 2014; Cruz y Rúa 2018). Los investigadores elaboraron una lista de 20 personas candidatas expertas que cumplieran con los siguientes criterios de argumentación (requisitos):

- Análisis teórico realizado por el experto (publicaciones en revistas científicas).

- Experiencia en consultorías en el área financiera.

- Experiencia en cargos gerenciales en el área de finanzas en sociedades anónimas (más de 3 años).

- $\quad$ Experiencia en cargos gerenciales en el área de finanzas en empresas cooperativas (más de 5 años).

- $\quad$ Experiencia en cargos gerenciales en el área de finanzas en empresas cooperativas (más de 5 años).

- Conocimientos acerca de procesos productivos alimentarios.

A continuación, se determinó el coeficiente de competencia según la siguiente fórmula:

K comp. $=1 / 2(K \mathbf{c}+\mathbf{K a})$ Coeficiente de competencia de cada experto

Donde:

Kc: Coeficiente de conocimiento

Ka: Coeficiente de argumentación

Para establecer el K comp. $=1 / 2(\mathbf{K c}+\mathbf{K a})$, se diseñó y aplicó una encuesta de estructurada con 15 preguntas (según los requisitos establecidos) para que, de forma individual, cada experto respondiera y otorgara a cada una 
de las preguntas, un valor, según el conocimiento personal. El coeficiente es el resultado del promedio de los valores que cada experto concedió en la respuesta a las preguntas. Para determinar el Ka, se suman de los valores del grado de influencia de cada uno de los criterios de argumentación con respecto a una tabla diseñada para ordenar los datos obtenidos de la encuesta (ver tabla 1).

El experto anotó el grado de influencia de cada uno de los criterios, según la escala: alta competencia, media competencia o baja competencia. Se suman los valores obtenidos y el resultado derivado representa el Ka obtenido por cada experto. Dados los coeficientes Kc y Ka, se calculan para cada experto y se obtiene el valor de K comp:

\begin{tabular}{|l|l|}
\hline La competencia del experto es ALTA & si K comp $>0.8$ \\
\hline La competencia del experto es MEDIA & si $0.5<\mathrm{K}$ comp $>=0.8$ \\
\hline La competencia del experto es BAJA & si $\mathrm{K}$ comp $<=0.5$ \\
\hline
\end{tabular}

\section{Tabla 1}

Influencia según criterios de argumentación

\begin{tabular}{|c|c|c|c|}
\hline $\mathrm{Cr}$ & $\begin{array}{l}\text { Alta } \\
\text { competencia }\end{array}$ & $\begin{array}{l}\text { Media } \\
\text { competencia }\end{array}$ & $\begin{array}{l}\text { Baja } \\
\text { competencia }\end{array}$ \\
\hline
\end{tabular}

Análisis teórico realizado por el experto (publicaciones en revistas científicas).

$\begin{array}{lll}0.3 & 0.2 & 0.1\end{array}$

Experiencia en consultorías en al área financiera.

$\begin{array}{lll}0.5 & 0.4 & 0.2\end{array}$

Experiencia en cargos gerenciales en el área de finanzas en sociedades anónimas (más de 3 años).

$\begin{array}{lll}0.05 & 0.04 \quad 0.03\end{array}$

Experiencia en cargos gerenciales en el área de finanzas en empresas cooperativas (más de 5 años).

$\begin{array}{lll}0.05 & 0.04 & 0.03\end{array}$

Conocimientos acerca de la aplicación de las normas internaciones de $\begin{array}{lll}0.05 & 0.04 & 0.03\end{array}$ información financiera (NIIF).

Fuente: Hernández y Pineda (2017). 
De esta forma y de un total de 20 especialistas, se eligieron siete expertos que obtuvieron la calificación de alta competencia. Es importante señalar que los expertos seleccionados no se conocieron entre sí. La tabla 2 muestra el perfil de los expertos seleccionados.

Tabla 2

Perfil de expertos

\begin{tabular}{|l|l|}
\hline Experta 1: & Gerente General de una empresa carnes procesadas. \\
\hline Experta 2: & Gerente de Finanzas en una empresa ganadera. \\
\hline Experto 3: & $\begin{array}{l}\text { Gerente Corporativo Financiero de una empresa de } \\
\text { productos alimentarios. }\end{array}$ \\
\hline Experto 4: & Gerente de Producción en una empresa agroindustrial. \\
\hline Experta 5: & $\begin{array}{l}\text { Tecnóloga de alimentos en una cooperativa de productos de } \\
\text { consumo humano. }\end{array}$ \\
\hline Experta 6: & $\begin{array}{l}\text { Gerente de Finanzas en una Cooperativa de productos } \\
\text { financieros. }\end{array}$ \\
\hline Experta 7: & $\begin{array}{l}\text { Auditora certificada en normas internacionales de información } \\
\text { financiera (NIIF). }\end{array}$ \\
\hline
\end{tabular}

La tabla 3 muestra los resultados obtenidos de los expertos seleccionados, según el alto nivel de competencia (Kcomp > 0.8). Esto permitió que cada especialista examinara el cálculo de análisis marginal y procediera a emitir un criterio técnico mediante una secuencia de validación establecida, con la finalidad de enriquecer el cálculo y con esto perfeccionarla para su posterior aplicación en la cooperativa de cogestión de productos cárnicos seleccionada.

Tabla 3

Cálculo del coeficiente de competencia de cada experto

\begin{tabular}{lllll}
\hline Experto & Kc & Ka & K comp & Calificación \\
\hline 1 & 1 & $0.5+0.3+4(0.05)=1$ & 1 & Alta \\
2 & 1 & $0.5+0.2+4(0.05)=0.9$ & 0,95 & Alta \\
3 & 0.9 & $0.2+0.5+0.04+3(0.05)=0.89$ & 0,895 & Alta \\
4 & 0.8 & $0.5+0.2+0.05+0.04+2(0.03)=0.85$ & 0,83 & Alta \\
5 & 0.9 & $0.2+0.5+4(0.04)=0.76$ & 0,86 & Alta \\
6 & 0.8 & $0.3+0.5+0.03+0.04+0.05+0.04=0.96$ & 8,88 & Alta \\
7 & 0.9 & $0.3+0.5+2(0.04)+0.05+0.04=0.97$ & 0,935 & Alta \\
\hline
\end{tabular}




\section{RESULTADOS}

\section{Definición de la identidad matemática básica}

Se tomaron en consideración, para definir la identidad matemática básica en la demostración del teorema aquí presentado, dos características que presenta el estado de resultados, tal como la frecuencia de los informes o periodicidad y la propia estructura del estado en cuestión. Especialmente en cuanto a la estructura del estado, se toman los cuatro componentes básicos, a saber: Ingreso por Ventas, Costos Totales, Gastos Totales, Utilidad o Pérdidas.

En cuanto a la periodicidad, la práctica contable-financiera generalizada de realizar análisis comparativos de periodos, también ha servido para establecer en este modelo, los momentos en el tiempo en el que se compararán con la ayuda de la identidad matemática. De tal manera, se pueden adicionar y correlacionar, además, los componentes básicos del estado de resultados, por medio de las fórmulas apropiadas que se proponen y que se derivan de la propia identidad principal. Es así que se recurrió a la formulación del rendimiento marginal y se adaptó a las necesidades de cálculo y a las diferentes condiciones citadas en los párrafos anteriores, de tal manera que la identidad básica es la siguiente, Pineda (2007):

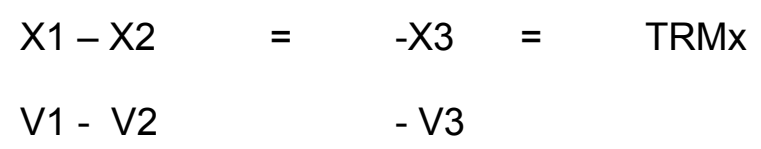

En donde,

"X" se refiere al componente básico del estado de resultados que se va a calcular, de tal forma que el (1) es el periodo más antiguo, contra el cual se realizará la correlación, que en este caso será el referente (que puede ser mes, trimestre, semestre, anual u otro) y el "2" es el periodo más reciente o de estudio.

"V" se refiere al nivel de ventas o de ingreso, según corresponda a la nomenclatura de la estructura del estado de resultados de que se trate. El "1" es el periodo más antiguo, contra el cual se realizará la correlación, que en este caso será el referente (que puede ser mes, trimestre, semestre, anual u otro) y el "2" es el periodo más reciente o de estudio. 
"-X3" es el resultado de la resta del periodo más antiguo de la variable de estudio ("1") o periodo de referencia y el periodo más reciente ("2"). Su resultado puede ser positivo o negativo y constituye el numerador de una fracción.

"-V3" Constituye el resultado de la resta del periodo más antiguo de ventas o ingresos ("1") o periodo de referencia y el periodo más reciente ("2"). Su resultado deberá ser negativo y es el denominador de una fracción de cálculo.

"TRMx" se denominaría Tasa de Rendimiento Marginal de la variable " $x$ ", siendo el resultado de la división entre "-X3" y "-V3"; el resultado puede ser negativo o positivo.

En el siguiente apartado, el lector podrá encontrar las fórmulas para las aplicaciones de cálculo que corresponden a cada componente básico del estado de resultados.

\section{Derivación de las fórmulas de los componentes del estado de resultados}

De la fórmula o identidad matemática (1), se derivan las fórmulas para cada uno de los tres componentes básicos restantes: costos totales, gastos totales y utilidad o pérdida neta. Se recordará que el ingreso o ventas es el elemento macro del estado de resultados del que se sustraen los gastos y los costos, por lo que su papel dentro del cálculo, quizás es el más relevante, pues la validez del cálculo depende de este.

En lo que respecta a la aplicación propiamente dicha, lo que se hace es sustituir la variable " $X$ " por las siguientes siglas. Se adjunta su significado.

"Ct", que corresponde a los costos totales.

"Gt", que corresponde a los gastos totales.

"U" o bien "P", que se refiere a la utilidad o pérdida según sea el caso. Se aclara que, en el caso de la pérdida, se respeta el signo negativo propio de tal situación. 
Las diferentes fórmulas serían las siguientes (Pineda, 2007):

Tasa de rendimiento marginal de los costos

$\underline{\mathrm{Ct} 1-\mathrm{Ct} 2}=\underline{\underline{\mathrm{Ct}} 3}=\mathrm{TRMc}$

$\mathrm{V} 1-\mathrm{V} 2 \quad-\mathrm{V} 3$

Tasa de rendimiento marginal de los gastos

$\underline{\mathrm{Gt}} 1-\mathrm{Gt} 2 \quad=\quad \underline{-\mathrm{Gt} 3}=\mathrm{TRMg}$

$\mathrm{V} 1-\mathrm{V} 2 \quad-\mathrm{V} 3$

Tasa de rendimiento marginal de las utilidades o pérdidas

$\underline{\mathrm{U} / \mathrm{P} 1-\mathrm{U} / \mathrm{P} 2}=\underline{-\mathrm{U} / \mathrm{P} 3}=\mathrm{TRMu} / \mathrm{p}$

Como se puede notar, la aplicación de la fórmula se efectuará para cada uno de los componentes. Se tiene, por tanto, una serie de resultados marginales que, en forma separada, pueden brindar una serie de datos del componente al que se refieren y en conjunto podrán reflejar de formas diversas su interacción.

\section{Parámetros marginales: su significado e implicaciones}

Se podrá comprender que como se está trabajando con indicadores marginales, se convierte en una necesidad el puntualizar parámetros que establezcan el significado de ese indicador. Estos resultados pueden ser considerados tasas o porcentajes, o bien, cifras que generalmente se encuentran entre el 0.00 y el 1.00 , en donde la posibilidad de que los resultados rebasen estos dos grandes límites es siempre latente.

Es así que se propone un conjunto de enunciados que indican los parámetros generales a utilizar y lograr así una adecuada interpretación de las cifras, lo que favorecerá el posterior análisis de los resultados, tanto de forma individual como en su conjunto, estos son (Pineda, 2007):

- Es deseable que la tasa de rendimiento marginal de los costos totales sea lo más cercana a "0.00" o con algún valor negativo. 
- Es deseable que la tasa de rendimiento marginal de los gastos totales sea lo más cercana a " 0.00 " o con algún valor negativo.

- $\quad$ Es deseable que la tasa de rendimiento marginal de las utilidades sea lo más cercana a "1.00", o bien, que supere la barrera marginal.

- Cuando una tasa de rendimiento marginal en los costos totales es negativa, lo que refleja es la tendencia a la mejora, ya sea por una influencia positiva en la reducción de estos, combinada con un proceso en el cual se diluyen por influencia de economías de escala.

- Si la tasa de rendimiento marginal de utilidades iguala o supera la barrera marginal, no implica que la unidad monetaria basada en 100 centavos deba ser mayor, sino que es causa de un crecimiento marginal negativo de alguna de las otras dos variables, sean los gastos o los costos y el posible impacto directo o indirecto de un crecimiento positivo de los ingresos o ventas.

- Es deseable el crecimiento marginal negativo de los costos en general, pues su impacto es más directo sobre la utilidad.

- $\quad$ El crecimiento negativo de los ingresos se convierte en la condición más adversa para el cálculo e interpretación de los datos con este modelo, además de lo inconveniente con respecto a la situación del negocio propiamente dicho.

Con los enunciados anteriores, se resumen algunas condiciones lógicas que corresponden a las diferentes interacciones entre los ingresos y egresos, así como otras relaciones existentes entre los componentes básicos del Estado de Resultados, sirviéndose de los indicadores que arrojan las fórmulas planteadas en el punto anteriormente citado. Entender los enunciados que llevan implícitos los parámetros generales se convierte en la base para la utilización de los indicadores calculados, dándole al analista la posibilidad de establecer correlaciones con datos históricos, preferiblemente en el largo plazo. Dichas comparaciones se puedan realizar con competidores del mismo sector de la industria.

Sin embargo, algunos elementos pueden ser comentados debido, principalmente, a su relevancia relativa dentro de un posible análisis e 
interpretación, tal como la reducción de los costos o de los gastos, que se pueden deber a políticas de racionalidad de estos. Por ejemplo, teniendo la posibilidad de diagnosticar por medio del proceso comparativo, la necesidad de establecer políticas en estos temas. Además, la decisión de contar con un proceso, por ejemplo, de una eficiente y oportuna administración de inventarios, sería una decisión importante, pues tendería a reducir los costos de almacenamiento y demás, lo que se transferiría directamente a las utilidades.

Sobre lo anterior, se considera relevante destacar que resulta en un mayor esfuerzo organizacional el incrementar en un determinado nivel las ventas que establecer una política clara de ahorro interno, ya que el ahorro se visualiza más fácilmente en la utilidad, que el incremento de las ventas para obtener ese mismo monto de ahorro. Por ejemplo, un porcentaje de utilidad sobre ventas de $25 \%$ se deberá colocar monetariamente cuatro veces lo que corresponde al monto ahorrado. Con lo anterior, no se debe de entender que el esfuerzo organizacional debe concentrarse en el ahorro, pues este elemento posee su propio nivel de importancia en comparación a la generación de ingresos por medio de la venta de bienes o servicios.

En consecuencia, las políticas, directrices o decisiones que se tomen están orientadas a maximizar la utilidad y a ser sostenible positivamente el negocio de la empresa. La combinación de factores, como resultado de un diagnóstico y análisis se convierte en el punto de partida para lograr esa sostenibilidad.

\section{INTERPRETACIÓN DE LOS ESTADOS DE RESULTADOS UTILIZANDO LOS INDICADORES MARGINALES}

Así pues, las diferentes combinaciones de lo que se podría llamar "la mezcla de decisión", pueden basar sus planteamientos en relación con indicadores que reflejen con propiedad el estado o situación de la empresa, línea de negocios, gerencia, etc. Se considera oportuno proponer un ejemplo de la división cárnica de la cooperativa de cogestión estudiada que favorezca el empleo de las herramientas y enunciados propuestos para ilustrar el uso e interpretación de los indicadores obtenidos, lo cual se ejemplificará basándose en la tabla 4 . 
Tabla 4

Estructura básica de un estado de resultados con aplicación del cálculo de las tasas de rendimiento marginal (Cifras monetarias en miles dólares)

\begin{tabular}{lcccc}
\hline \multirow{2}{*}{ RUBRO/ MES } & \multicolumn{2}{c}{ AÑO } & \multicolumn{2}{c}{ AÑO } \\
& ABRIL & MARZO & ABRIL & MARZO \\
\cline { 2 - 5 } & 15000 & 13500 & 12200 & 10750 \\
VENTAS & 10000 & 8750 & 7800 & 6800 \\
COSTOS TL & 2000 & 1850 & 1850 & 1650 \\
GASTOS TL & 3000 & 2900 & 2550 & 2300 \\
UTILIDAD & & & & \\
& 0.833 & & 0.690 \\
TRMc & 0.100 & \multicolumn{2}{c}{0.138} \\
TRMg & 0.067 & \multicolumn{2}{c}{0.172} \\
TRMu/p & 1.000 & \multicolumn{2}{c}{1.000} \\
Sumatoria & & & \\
\hline
\end{tabular}

Para ilustrar la aplicación del cálculo se presenta de seguido el ejercicio para determinar la tasa de rendimiento marginal de las utilidades o pérdidas de la Cooperativa de Cogestión de productos cárnicos estudiada, con base a la tabla 4, correspondiente a los meses de marzo y abril 2015- 2016.

\section{Tasa de rendimiento marginal de las utilidades o pérdidas}

$\underline{\mathrm{U} / \mathrm{P} 1-\mathrm{U} / \mathrm{P} 2}=\underline{-\mathrm{U} / \mathrm{P} 3}=\mathrm{TRMu} / \mathrm{p}$

$\mathrm{V} 1-\mathrm{V} 2 \quad-\mathrm{V} 3$

Sustituyendo los valores:

$\underline{2900-3000}=\underline{-100}=0,067=\mathrm{TRMu} / \mathrm{p}$

$13500-15000-1500$

De igual manera, la aplicación de las diferentes fórmulas es similar, variando los datos según sean los costos, gastos o ingresos. Ahora bien, teniendo los indicadores ya calculados del ejemplo anterior, se pueden hacer los siguientes enunciados:

Se requieren dos periodos para realizar el cálculo de los diferentes indicadores. El hecho de que se realicen dos cálculos, uno para cada año, es 
solamente para establecer una comparación entre indicadores en meses iguales, pero momentos diferentes.

En la última línea de la tabla, indicado por el rubro "sumatoria" se puede visualizar lo que en puntos anteriores se mencionaba: los componentes llamados costos totales, gastos totales y las utilidades o pérdidas sumadas tienen el mismo resultado que los ingresos o ventas; de igual manera, la sumatoria de los indicadores.

Derivado del punto anterior, la condición de la línea final llamada "sumatoria" brinda una interpretación como: "De una unidad monetaria cualquiera, " $x$ " cantidad de centavos se destinan a los costos, " $x$ " cantidad se consumen en gastos $y$ " $x$ " cantidad quedan como utilidad o pérdida".

Entonces, para el caso del ejemplo de la tabla 4, para el mes de abril del 2015 , de una unidad monetaria de ingresos por ventas, $83.3 \%$ se consumieron en costos totales; $10 \%$ se consumieron en gastos totales y $6.7 \%$ quedaron como utilidad.

De estos datos se podría indagar si el porcentaje de utilidad que se está empleando para calcular el valor de las mercancías que se colocan para la venta es el correcto; si las negociaciones con los proveedores son ventajosas o pueden ser mejor aprovechadas, ya que de no ser así, el costo de las mercaderías sería alto, debiendo sacrificar rentabilidad para ser competitivos. Por ejemplo, si hay una adecuada rotación de los inventarios o existen productos con una baja rotación y se tiene una alta existencia en bodegas, elemento que incrementa los costos de almacenamiento; evaluar si las políticas de control de gastos están funcionando o si se deben replantear; tomar en cuenta si la contabilidad está por error transfiriendo rubros de gastos como costos, debido a diferencias que pueden ser irregulares.

Con respecto a la comparación de periodos iguales, pero en años diferentes, como el caso del ejemplo citado, se puede relacionar el caso de la tasa de rendimiento marginal de los costos, en donde este indicador varía del año 2015 al 2016. Asimismo, en el año 2016, este rubro consumió 83.3\% de una unidad monetaria de ingresos. La explicación probable sobre este punto es que la dependencia matemática que tienen los costos totales con respecto a las ventas es significativa, pues dentro de los costos está considerado el valor de la mercadería o del inventario. Es así que, a mayores 
ventas, mayor es la cantidad del valor de mercadería vendida como costo. Como se indicó en párrafos anteriores, para el caso del ejemplo se podría verificar las negociaciones con los proveedores y el porcentaje de utilidad en el cálculo del precio de venta de los productos, pues quizás la rentabilidad general de este negocio no satisface las expectativas de la gerencia o accionistas, así como la valoración de otros elementos ya mencionados.

Para el segundo punto del análisis en relación con la comparación de los costos de un año y otro, se puede verificar que la diferencia radicó en que la relación de las ventas o ingresos y los costos fue mucho más ventajosa en el 2015 que en el 2016, pues la diferencia del crecimiento de los meses de cada uno de los años es similar. De aquí que la TRMc resulta más eficiente en el 2015 que en el 2016 , elemento que se refleja también en la TRMu/p.

De igual manera, al analizar los gastos totales, se puede comparar el grado de eficiencia relativa a la gestión y control del gasto, aspecto que dará pie a un proceso permanente de evaluación de las acciones estratégicas, políticas y procedimientos vinculados con este tema.

Bajo el escenario de que los niveles de ventas tienden a ser similares y a reducir las eficiencias o rentabilidad, con el estudio de estos indicadores puede también llegar a cuestionarse la propia eficiencia de la gestión de ventas, además de las consideraciones citadas. La verificación de la labor de crédito y cobro con énfasis en sus estrategias de cobro, fijación de límites crediticios y selección de cartera de clientes, que ayuden además a reducir el promedio de recuperación del efectivo que está como crédito; también, si el crecimiento de las ventas, se debe a efectos inflacionarios, que pueden visualizarse con los indicadores, situación debido a que se está vendiendo la misma cantidad de unidades de productos pero a precios mayores, causado por el efecto citado.

Con los puntos anteriores, se puede brindar una idea del tipo de reflexiones, resultado de un análisis tomando como herramienta los indicadores marginales, ofreciendo al técnico en análisis la oportunidad de realizar diferentes relaciones numéricas. Cuando la empresa posea suficiente experiencia en la utilización de indicadores marginales, tendría la posibilidad de mejorar sus análisis con relación a su entorno. 
La alta gerencia de la cooperativa analizada podría, a mediano plazo, establecer sus metas en función de los indicadores, además de las metas absolutas o en unidades de producción o monetarias. Además, la oportunidad que se deja patente en esta investigación, pero que no se desarrolla, pues no forma parte de sus objetivos, es la de especializar el uso de los indicadores marginales, de tal manera que se puedan estudiar las subpartidas que componen cada uno de los componentes básicos del estado de resultados. La idea es puntualizar cada vez más la información de los rubros esenciales de dichas partidas, teniendo un mayor o mejor control de las diferentes variables, cerrando el círculo de análisis para que, cada vez más, la interpretación de los estados de resultados tienda a ser más exacta, tecnificando su aplicación por medio de una ciencia precisamente exacta, la matemática.

\section{Limitaciones del modelo de cálculo}

Aún con las bondades de un sistema de cálculo, las variables o circunstancias que rodean a una gestión de negocios son muy complejas, el concepto conocido como ceteris paribus tiende a ser en muchos modelos de cálculo, un elemento sustancial que contribuye solamente a la comprensión teórica de conceptos.

El presente modelo prescinde en buena medida de la condición mencionada en el párrafo anterior; sin embargo, existe una limitación en el cálculo que tiene plena relación con la realidad de los negocios. Es la situación del denominador de la identidad (1) y las otras fórmulas.

El planteamiento del modelo ha hecho énfasis en que esta parte es la que favorece o no la vigencia del teorema; la relación de la resta que se hace de las ventas o ingresos del periodo de referencia (V1) menos el periodo de estudio (V2) debe ser negativa (-V3) para que el cálculo sea efectivo. En otras palabras, las ventas o ingresos del mes actual o de estudio deben ser mayores, en cualquier cifra, que las ventas o ingresos del mes anterior o de referencia.

Si V3, es positivo, al efectuar la división de -X3 entre V3, ocasionado por un decrecimiento de las ventas o ingresos del periodo actual con respecto al anterior, el resultado de la Trox será negativo en los diferentes cálculos de los componentes básicos del Estado de Resultados, presentando una 
imposibilidad de análisis y, por ende, de interpretación. Si bien es cierto que, bajo condiciones normales, (-V3) alguno de los indicadores TRMct, TRMgt o TRMu/p pueden presentarse en forma negativa, la interpretación siguiendo los enunciados o parámetros generales planteados tiene sentido lógico y una explicación matemática coherente. La situación irregular que se ha descrito carece según la experimentación de éstas condiciones. A pesar de la limitación planteada, un desarrollo posterior de este modelo propuesto, podría cambiar el panorama descrito, sin que se dejen de lado los conceptos financiero-contables básicos que la presente investigación toma, acepta y emplea para dar sentido lógico a los datos obtenidos.

\section{CONCLUSIONES}

El análisis vertical y horizontal se utiliza principalmente por el uso extendido y de plena aceptación que tiene entre los especialistas financieros. Además, supone el contraste más cercano a la técnica empleada con el análisis marginal que se ha propuesto. Así las cosas, dentro del análisis horizontal y vertical, para corroborar los datos del análisis marginal, se tendrían que diseñar al menos 4 tablas, dos por cada sección del análisis.

Lo anterior se debe a que se están corroborando dos meses seguidos del mismo año, pero también, dos meses de años diferentes. Mientras que, en el cálculo del modelo marginal propuesto, la función de correlación que precisamente brinda el teorema de cálculo, favorece el contraste de diferentes datos, pero cuya correlación debe guardar significancia en tiempo o en espacio, fundamentalmente para que la asociación de datos y sus resultados tengan sentido y valor. Una vez aclarada la diferencia de presentación, asociación y correlación de datos, se empleará el ejemplo de estructura básica del estado de resultados de la tabla 4, para poder contextualizar numéricamente, el aporte tradicional del análisis horizontal y vertical.

Dentro de la sección horizontal del análisis, donde se contrastan los meses de marzo y abril del 2015, es justo partir de que existe utilidad, pero la variación de esta entre un mes y otro no es significativa, pues solamente crece en 100 dólares, correspondiente a un crecimiento del $3.45 \%$. Al corroborar los gastos y costos, existe una relación similar de un mes a otro, por lo que el esfuerzo de ventas, donde el crecimiento es $11.11 \%$, debería 
ser mayormente profundizado, beneficiándose así de las economías de escala, propiciando una reducción eventual del gasto.

En lo que respecta a la comparación del mes de abril del 2016 y del 2015, en ambos momentos hay utilidad, solamente que la de abril del 2015 es mayor, correspondiendo a $17.65 \%$ más. Al mantenerse controlado el gasto, el aumento en las ventas (22.95\%) absorbió el también esperado incremento en los costos, lo cual es inevitable por el costo precisamente de la mercadería que se va a comercializar, así como otros aspectos asociados, por lo que aun cuando la diferencia de abril del 2015 con respecto al mes anterior del mismo año, no fue tan significativa, al contrastar este mes con el mismo del año anterior, el crecimiento favorable es evidente.

En cuanto a la sección vertical del análisis, la acción matemática es la de establecer la relación de los costos, gastos y utilidad con respecto a las ventas. Quizás la relación utilidad sobre ventas es la que primeramente se desea obtener en los resultados mensuales, o bien, de acuerdo al periodo en el cual se enmarque el análisis. En la comparación entre marzo y abril del 2015, tal y como se indicó, existe una utilidad en cada mes, lo que también se refleja en la relación con respecto a las ventas de $20.00 \%$ en abril y $21.48 \%$ en marzo, donde no solamente hay una desaceleración de un crecimiento relativo, sino que se refleja un crecimiento negativo de la utilidad sobre ventas entre un mes y otro.

Ahora bien, en la relación de datos de abril del 2016 y del 2015, el porcentaje de utilidad sobre ventas fue $20.00 \%$ y $20.90 \%$ respectivamente. Nuevamente se puede establecer que, aun cuando existe una utilidad respectiva en cada mes o momento, esta tiene un crecimiento negativo entre los periodos comparados. Por ello, el esfuerzo de ventas y la correspondiente transferencia de valor que deben propiciar la utilización de los costos y de los gastos no se ven debidamente reflejados en la utilidad, especialmente del mes de estudio que es el de abril del 2015.

Como se aprecia, utilizar el modelo de análisis marginal desde la gerencia estratégica le da la oportunidad a los tomadores de decisiones económicas, de puntualizar y discernir en qué fase del ejercicio gerencial se deben tomar las decisiones para enrumbar el negocio, corregir un objetivo, formular una estrategia de mercado, variar el precio del servicio o producto, entre otros. Todo esto con el fin de darle valor a la actividad de 
emprendimiento, siendo la agilidad, la eficacia y la exactitud de los diagnósticos una de las piezas fundamentales para aprovechar las oportunidades diversas que ofrecen los mercados, o bien, el propio sector industrial al que se pertenece.

Por otra parte, con el diagnóstico interpretado en el justo a tiempo, logrando reforzar la estrategia o variarla para maximizar sus efectos, es como se logra la maximización también de las utilidades, siendo beneficioso para los asociados de la cooperativa, independientemente del sector o agrupación al que pertenezcan, así como de las cuantías de los aportes de capital invertidos; ello significa que cualquier posibilidad de mejoría en la gestión de la organización posee un efecto multiplicador a nivel social, de desarrollo y de redistribución de la riqueza, ya que el impacto sobre las familias de los asociados es directa, mitigando el problema de desempleo que presenta el país (Hernández, 2015).

De igual forma, la influencia indirecta de ese fenómeno multiplicador podrá verse manifestado en el entorno local de las familias; es decir, en las comunidades anfitrionas, mediante el consumo, el ahorro, la cancelación de tributos, cuyas ramificaciones socioeconómicas son sin duda de gran valor estratégico para todo emprendimiento cooperativo de cogestión, en el marco de las externalidades cooperativas positivas.

En este contexto, el sector cooperativo, ha sido una respuesta a los diferentes modelos de desarrollo empresarial, diferenciándose de las empresas privadas. Estas últimas persiguen obtener el máximo beneficio para los interesados financieros o stakeholders de la empresa. Las cooperativas son organizaciones que fomentan la democratización y empoderamiento de la propiedad productiva de una zona, región o país. A nivel internacional, estas organizaciones, que son generalmente de un carácter diferenciador de constitución privada, pero de interés público, se denominan con diferentes patronímicos según sea su conformación creacional en diferentes países como organizaciones que pertenecen a sectores tales como: economía social, economía solidaria, tercer sector, economía popular, organizaciones de desarrollo social, sector de la economía laboral entre otras apelaciones. Según la Organización de Naciones Unidas, más de tres mil millones de personas obtienen su sustento diario por medio del sector cooperativo. Es decir, casi la mitad de la población mundial de una u otra manera está integrada al sector cooperativo tan dinámico de la sociedad (ONU, 2014). 
En Costa Rica, el cooperativismo representa el 39.9\% de la Población Económicamente Activa (PEA) y $4.96 \%$ del Producto Interno Bruto (PIB). En contraparte con la apertura comercial experimentada por Costa Rica, el sector se ha mantenido en un promedio de 372 cooperativas desde los años ochenta a la fecha, pero no así su número de miembros, el cual se incrementó en un 442\%, equivalente a 837335 mil asociados (Programa Estado de la Nación, 2012). Ello, ha contribuido a disminuir el impacto del desempleo en el país.

\section{REFERENCIAS}

Amaro, Y. (2012). El análisis marginal. Argentina: Buyatti.

Cabero, J. \& Infante, A. (2014). Empleo del método Delphi y su empleo en la investigación en comunicación y educación. Edutec. Revista Electrónica de Tecnología Educativa, 48. Recuperado de http://www. edutec.es/revista/index.php/edutec-e/article/view/187

Campos, V., Melián, A., y Sanchis, J. (2014). El método Delphi como técnica de diagnóstico estratégico. Estudio empírico aplicado a las empresas de inserción en España. Revista Europea de Dirección y Economía de la Empresa, 23(2), 72-8.

Cohen, R. (2011). Análisis marginal. Argentina: Facultad de ingeniería, Universidad de Buenos Aires.

Cortés, M. E. e Iglesias, M. (2005). Generalidades sobre Metodología de la Investigación. México: UNACAR.

Cruz, M. y Rúa, J. (2018). Surgimiento y desarrollo del método Delphi: una perspectiva cienciométrica. Revista de Bibliotecología y Ciencias de la información, 71(71), 1-18. doi: 10.5195/biblios.2018470

David, F. y David, F. (2017). Conceptos de administración estratégica. México: Pearson.

Drucker, P. (2004). What makes an Effective Executive. En Harvard Business Review, July-August. EE.UU. 
Evans, E. (2017). Análisis Marginal: Un Procedimiento Económico para Seleccionar Tecnologías o Prácticas Alternativas. Florida: Universidad de Florida.

Gitman, L., Berk, J., DeMarzo, P., y Benjamin, F. (2018). Principios de administración financiera 1, 2. México: Pearson.

Gitman, L. (2010). Principios de Administración Financiera. México: Pearson.

Golpe, A. (2016). La Gestión Estratégica de Costos, como instrumento de planificación social. Caso: Proyecto centro cooperativo de producción en Uruguay. XXIII Congresso Brasileiro de Custos - Porto de Galinhas, PE, Brasil, 16 a 18 de novembro de 2016.

Gomero, N. y Montes, E. (2011). Emprendurismo y análisis marginal de las inversiones. Revista de la Facultad de Ciencias Contables, 18(35), 173-182.

Griffin, R. (2011). Administración. México: Cengage Learning.

Hardford, J., Berk, J., y DeMarzo, P. (2010). Fundamentos de finanzas corporativas. México: Pearson.

Hernández, G. (2013). Gestión de calidad en las empresas turísticas sostenible. Costa Rica: EUNED.

Hernández, G. (2015): Capítulo 1. Globalización y desempleo. Las cooperativas de trabajo en América Latina, p. 419-443. Argentina: Ediciones DelRevés.

Hiller, F. y Hiller, M. (2008). Métodos cuantitativos para la administración. México: McGraw Hill.

Kline, R. (1998). Principles and Practice of Structural Equation Modeling. New York: The Guilford Press.

Lambreton, V. y Garza, G. (2016). Información financiera en los negocios. México: Pearson. 
Landeta, J. (2002). El Método DELPHI. Barcelona: Ariel.

Mankiw, N. (2015). Principios de Economía. México: CENGAGE Learning.

Organización de las Naciones Unidas [ONU]. (2014). Estudio mundial de las cooperativas. New York: ONU.

Pineda, S. (2007). Aplicación de un modelo de análisis para Estados de Resultados, utilizando la teoría de Análisis Marginal. Tesis de Licenciatura en Administración de Empresas con Énfasis en Banca y Finanzas. Universidad Estatal a Distancia, Costa Rica.

Porter, M. (2009). Estrategia competitiva: técnicas para el análisis de la empresa y sus competidores. México: Ediciones Pirámide.

Programa Estado de la Nación. (2012). IV Censo Nacional Cooperativo. Costa Rica: INFOCOOP.

Ross, S., Westerfiel, R., y Bradford, J. (2010). Fundamentos de finanzas corporativas. México: McGraw Hill.

Rufasto, A. (2007). La perspectiva de la Empresa. Recuperado de www. geocities.com/arufast-http://rufasto.tripod.com

Sanchis, J. y Campos, V. (2006). La Dirección Estratégica en la Economía Social: utilización de herramientas de análisis estratégico en las Cooperativas. Revista de Economía Pública, Social y Cooperativa, (59), 237-258.

Thomas, W. y Hunger, D. (2013). Administración estratégica y política de negocios. Hacia la sostenibilidad global. México: Pearson.

Weston, F. y Brigham, E. (1994). Fundamentos de Administración Financiera. México: McGraw Hill. 
